\title{
Epidemiological Studies on Early Blight of Potato under Climate Change and its Co-Relation with Disease Severity
}

\author{
Praveen Kumar, S.K. Biswas*, Virendra Kumar and Kishan Lal \\ Department of Plant Pathology, C.S.A. University of Agriculture and Technology, \\ Kanpur-208002, India \\ *Corresponding author
}

\section{A B S T R A C T}

Occurrence and severity of Early blight of potato observed that the disease occured every year at Kanpur and Faizabad district of Utter Pradesh. It has observed that the disease was

\section{Keywords}

Early blight,

Disease severity,

Weather

parameters,

Climate change

Article Info

Accepted:

17 April 2017

Available Online:

10 May 2017

first appeared during $2-3^{\text {rd }}$ weeks of December at Kanpur and $3^{\text {rd }}$ weeks of December to $1^{\text {st }}$ weeks of January at Faizabad district of Uttar Pradesh at the maximum temperature range $17-23^{0} \mathrm{C}$, minimum temperature $1.8-7.8^{\circ} \mathrm{C}$, relative humidity $46-93 \%$, and $0-7.2$ sun shine hours. The maximum severity with 28.20 per cent was noted on 24 January, 2012 after $40^{\text {th }}$ days of first appearance of disease at the maximum temperature $23.0^{\circ} \mathrm{C}$ and minimum temperature $5.6^{\circ} \mathrm{C}$, relative humidity ranges $54-85 \%$ and sun shine hours 6.9 whereas, in the year 2013, maximum severity of 14.11 per cent on 23 January, 2013 after 41 days first appearance of disease at temperature range between $24.8^{\circ} \mathrm{C}$ to 8.8 , relative humidity $62-90 \%$ and 7.0 sun shine hours, respectively. It has also found that infection rate increases with occurrence of rainfall, resulted high relative humidity and low sunshine hours which are optimum for growth and sporulation of the pathogen during both the year. Similar observation has also been recorded at Faizabad district of Uttar Pradesh. The corelation between disease severity with temperature, relative humidity and sunshine hours were calculated by standard statistical calculation and the results showed that disease severity co-relate negatively with temperature $(-0.17845,-0.51214)$ and sunshine hours ($0.04185,-0.70934)$ and positive correlation with relative humidity $(0.638215,0.149054)$ at Kanpur during 2011 -2012. Similar observation has also been recorded in both the year at Faizabad.

\section{Introduction}

Potato (Solanum tuberosum L.) is the most useful vegetable crop in the world, belonging to the family Solanaceae. It is grown in about 150 countries of the world and is consumed by over a billion of peoples across the globe, of which half are in the developing countries. The United State Organization declared the year 2008 as "International Potato Year" (Shalbala and Pathak, 2008). The global area under potato production during 2011 was about 19.33 million hectares with a total production of about 321 million tonnes and having productivity of about 17.25 tonnes per hectares.

The intensive and extensive cultivation of potato even under the most favorable environmental conditions, the state failed to provide significant strides in potato yields, because of a number of production 
constraints. Among them, early blight caused by Alternaria solani, is noted worthy and has been taking heavy loss of the produce.

In Indo-Gangetic plains, depending upon prevailing weather condition, the early appearance of disease may seriously damage the potato crop and causing worry in the midst of potato growers. Yield loss estimates resulting from foliar damage incited by early blight on potato vary by location, cropping season, cultivar, and the stage of potato maturity. The yield reductions of 5 to $40 \%$ have been reported in Israel (Rotem and Feldman, 1965) and 20 to $30 \%$ in the USA (Christ and Maczuga, 1989; Shtienberg et al., 1990). Early blight may also cause dry rot of tubers, reducing both the quantity and quality of marketable tubers (Nnodu et al., 1982).

Management of the disease can be done through host resistance, cultural adjustments biological and use of fungicides. But there is no doubt that chemicals are best for management of disease. But continuous use of chemicals is not economical and eco-friendly. Therefore, the management practices should be focus on preventive or prophylactic measure that will reduced the initial inoculums resulted slow development of disease. Disease forecasting, may reduce the use of fungicides to minimize the development of disease under changing climatic condition. Therefore, there is urgent need to develop some kind of disease forecasting/warning services for predicting the time of appearance of disease and optimizing use of fungicides without risking the crop and human health. Hence, the study was taken in the present investigations.

\section{Materials and Methods}

\section{Survey for ascertaining the prevalence and severity of disease}

Survey was conducted at Vegetable Research Farm and Department of Plant Pathology,
Chandra Shakhar Azad University of Agriculture and Technology Kanpur and farmers field at the vicinity area of Kanpur and Faizabad of Utter Pradesh, during 20112012 and 2012-2013, in order to find out the prevalence and intensity of early blight of potato caused by Alternaria solani. The weather parameters which influenced the development of disease are also collected to correlate between weather parameters and disease intensity.

\section{Weather data}

The daily weather data was collected from weather station Department of Agronomy, C. S. Azad University of Agriculture and Technology Kanpur, Department of Meteorology, N. D. University of Agriculture \& Technology, Faizabad, India.

\section{Measurement of disease severity}

The crop was regularly observed for the first appearance of the disease. Progress of the severity of disease was also recorded daily. The observations on date of first appearance and maximum severity per cent of each disease were recorded separately. Disease severity was recorded using a score chart consisting of 0-9 scale as described by Malcolimson, 1976. Fifty leaves were randomly selected from the field for measurement of disease severity. The leaves with $1-9 \%$ infection received $1,10 \%$ infection received $2,11-25 \%$ infection received $3,26-$ $40 \%$ infection received $4,41-60 \%$ infection received $5,61-70 \%$ infection received 6,71 $80 \%$ infection received $7,81-90 \%$ infection received 8, 91-100\% infection received 9 (Malcolimson, 1976). The disease severity of individual plants was calculated by following formula

Disease severity PDI=

Sum of numerical rating

Total number of leaves examined $\mathrm{x}$ maximum rating $\mathrm{x} 100$ 
Correlation of disease severity with temperature, relative humidity and sunshine hours

The correlation between disease severity with temperature, relative humidity and sunshine hours were calculated by standard statistical calculation.

\section{Results and Discussion}

\section{Survey and severity of early blight of potato}

The observations on occurrence and severity of disease of early blight of potato observed that the disease occur every year at Kanpur and Faizabad of Uttar Pradesh during rabi season, 2011-12 and 2012-13.

\section{At Kanpur}

The data presented in the tables 1 and 2, showed that the first appearance of early blight was observed on $16^{\text {th }}$ December 2011 with the value of 0.55 per cent at the maximum temperature 21.0, minimum temperature 1.8, maximum relative humidity $93 \%$, minimum relative humidity $39 \%$ and 7.2 sun shine hours and maximum severity of 28.20 per cent was noted on 24 January, 2012 after $40^{\text {th }}$ days of first appearance of disease at the maximum temperature $23.0^{\circ} \mathrm{C}$ and minimum temperature $5.6^{\circ} \mathrm{C}, \quad$ relative humidity ranges $54-85 \%$ and sun shine hours 6.9 (Table 1). From the table 1, it is cleared that the infection rate is suddenly increased from 23.12.11 to 24.12.11 and 28.12.11 to 29.12 .11 which might be due to occurrence of rainfall, resulted high relative humidity and low sunshine hours which are optimum for growth and sporulation of the pathogen. On the other hand, result enumerate in table 2 showed that during second year, the first appearance of early blight was observed on dated $14^{\text {th }}$ December, 2012 with the value of 0.14 per cent at the $17.06^{\circ \mathrm{C}}$ maximum temperature and 7.8 minimum temperature, 97 per cent maximum and 79 percent minimum relative humidity and 0 sun shine hours and maximum severity of 14.11 per cent on 23 January, 2013 after 41 days first appearance of disease at temperature range between $24.8^{\circ} \mathrm{C}$ to 8.8 , relative humidity 62 $90 \%$ and 7.0 sun shine hours, respectively. From table 2, it is cleared that during 201213 , cloud covered continuously at frequent interval of date but light rainfall occurs on $8^{\text {th }}$ and $9^{\text {th }}$ January, considering favorable condition for development of disease, resulted increased infection rate.

\section{At Faizabad}

Two years data of Faizabad district presented in tables 3 and 4 showed that the first appearance of early blight was appeared on $18^{\text {th }}$ December 2011 with the value of 0.42 per cent at maximum temperature $23.5^{\circ} \mathrm{C}$ and minimum temperature $6.0^{\circ} \mathrm{C}$, maximum relative humidity $88 \%$, minimum relative humidity $46 \%$, and sun shine hours 7 . The maximum disease severity was recorded on $24^{\text {th }}$ January, 2012 with the value of $29.13 \%$ per cent at maximum temperature $24^{0 \mathrm{c}}$, minimum $5^{0} \mathrm{C}$, maximum relative humidity $90 \%$, minimum $59 \%$ and 7.0 sun shine hours (Table 3). From the table 3 it is also cleared that the infection rate was suddenly increased from $1-2^{\text {nd }}$ January, 2011 which might be high relative humidity and continuous cloudiness from 28.12.11 to 1.01.2012. During 2012-13, it was evident from table 4 that the first appearance of disease was occur on dated $16^{\text {th }}$ December, 2012 with the value of 0.23 per cent when environmental factors were $23.4^{\circ} \mathrm{C}$ maximum temperature and 7.8 ${ }^{\circ} \mathrm{C}$ minimum temperature, $98 \%$ maximum relative humidity and $67 \%$ minimum relative humidity and 0.0 Octa sun shine hours. The maximum severity of disease 31.23 per cent was noted on $21^{\text {th }}$ January, 2013 after 38 days from first appearance of disease at the maximum temperature $25^{\circ} \mathrm{C}$ and minimum 
$0.7{ }^{\circ} \mathrm{C}$, maximum relative humidity $89 \%$ and minimum $38 \%, 7$ sun shine hours, respectively. From table 2 it is cleared that infection rate rapidly increased from $7-8^{\text {th }}$ January which might be due to favorable environment factors indicating cloudiness from $6-8^{\text {th }}$ January, slight rainfall $(3.2 \mathrm{~mm})$ on $8^{\text {th }}$ January followed by high relative humidity (91 per cent) and low sunshine hours.

\section{Correlation between disease severity with temperature, relative humidity and} sunshine hours

\section{At Kanpur}

The correlation of disease severity with temperature, relative humidity and sunshine hours at Kanpur during 2011-12 has been calculated and data presented in the tables 5 and 6. It has been found that the disease severity showed the negative correlation with temperature (0.3664), (0.72) and positive correlation with relative humidity $(-0.493)$, (0.042 ) and the partially significant and negative correlation with sunshine hours (0.709). During 2012-13, similar observations have also been found from Kanpur area during 2012 - 13. The data from the (Table 6) showed that the negative co-relation of disease severity with temperature $(0.55447),(0.19292)$ positive co-relation with relative humidity $(0.00905),(-0.37277)$ and negative co-relation with sunshine hours (0.5233 ) (Figs. $1 \& 2$ ).

\section{Faizabad}

The data on disease severity, temperature, and relative humidity and sunshine hours are also collected from Farmer's field of Faizabad district during 2011-12 and 2012-13 and corelation study was done between them. The data presented in the table 7 showed that disease severity co-relate negatively with temperature and sunshine hours, representing the value (0.27322), (0.54021) and (0.59335), (-0.3411) respectively, but positively co-relate with relative humidity (0.61419), during 2011-12 and similar observations have also been found during 2012-13. The data presented in the table 8 that disease severity showed the partially significant and negative correlation with temperature $(0.48844),(0.47148)$ partially significant and positive correlation with relative humidity(0.023257), (-0.25609) nonsignificant and negative correlation with sunshine hours (-0.50429) (Figs 3\&4).

Environmental factors such as temperature, wetness duration, relative humidity (moisture), sunshine hours, rainfall affect the development of early blight on potato were reported by several workers (Adams and Stevenson, 1990; Harrison et al., 1965; Vloutoglou and Kalogerakis, 2000). Vander Walls et al., (2001) also reported that alternating low and high humidity conditions have also been shown to favour disease development.

Singh (2007) reported that the disease becomes serious when the season begins with abundant moisture or frequent rains followed by warm and dry weather. $\mathrm{He}$ also reported that higher mean temperatures $\left(19.2-31.1^{0} \mathrm{C}\right)$, frequent rains but shorter duration of relative humidity above $80 \%$, the absence of dew during most part of the season, longer photoperiod and prolonged senescence of the plants are related to low sporulation, restricted size of lesions, and moderate intensity of the disease.

Troutt and Levetin (2001) also found that there was a strong positive correlation between spore concentration and temperature. 
Table.1 Disease severity of early blight of potato (Alternaria solani) during 2011-2012 at Kanpur

\begin{tabular}{|c|c|c|c|c|c|c|c|c|c|c|}
\hline \multirow[t]{2}{*}{ Date } & \multirow{2}{*}{$\begin{array}{c}\text { Disease } \\
\text { severity }(\%)\end{array}$} & \multicolumn{2}{|c|}{ Temp. } & \multicolumn{2}{|c|}{ RH. (\%) } & \multirow[b]{2}{*}{$\begin{array}{c}\text { Sun } \\
\text { Shine }\end{array}$} & \multirow[b]{2}{*}{$\begin{array}{c}\text { Evaporati } \\
\text { on }\end{array}$} & \multirow[b]{2}{*}{ Rainfall } & \multicolumn{2}{|c|}{ Soil Temp. } \\
\hline & & Max & Min & Max & Min & & & & Max & Min \\
\hline 16.12 .2011 & 0.55 & 21.0 & 1.8 & 93 & 39 & 7.2 & 1.0 & 0.0 & 8.0 & 20.0 \\
\hline 17.12 .2011 & 0.60 & 22.2 & 1.8 & 97 & 51 & 00 & 1.0 & 0.0 & 8.0 & 21.0 \\
\hline 18.12 .2011 & 0.86 & 23.0 & 4.4 & 94 & 37 & 5.7 & 1.0 & 0.0 & 9.4 & 22.5 \\
\hline 19.12 .2011 & 1.32 & 24.0 & 5.4 & 97 & 48 & 4.1 & 1.0 & 0.0 & 10.0 & 21.6 \\
\hline 20.12 .2011 & 1.98 & 24.0 & 4.6 & 95 & 48 & 6.3 & 1.0 & 0.0 & 10.4 & 22.0 \\
\hline 21.12 .2011 & 1.45 & 24.0 & 8.2 & 94 & 52 & 4.1 & 1.0 & 0.0 & 12.2 & 18.5 \\
\hline 22.12 .2011 & 2.45 & 16.4 & 14.2 & 98 & 91 & 0 & 1.0 & 4.7 & 16.0 & 15.0 \\
\hline 23.12 .2011 & 3.25 & 17.0 & 10.6 & 98 & 87 & 0 & 1.0 & 44.4 & 14.0 & 15.0 \\
\hline 24.12 .2011 & 7.05 & 20.8 & 10.8 & 93 & 74 & 0 & 1.0 & 0.3 & 13.5 & 21.4 \\
\hline 25.12 .2011 & 7.85 & 20.2 & 9.2 & 97 & 69 & 0 & 1.0 & 0.0 & 13.4 & 20.2 \\
\hline 26.12.2011 & 8.11 & 18.4 & 9.2 & 97 & 82 & 0 & 1.0 & 0.0 & 14.4 & 20.0 \\
\hline 27.12 .2011 & 8.46 & 15.4 & 10.2 & 98 & 93 & 0 & 1.0 & 0.0 & 15.0 & 15.0 \\
\hline 28.12 .2011 & 8.50 & 18.2 & 12.2 & 95 & 77 & 0 & 1.0 & 4.8 & 14.6 & 19.0 \\
\hline 29.12 .2011 & 10.23 & 18.6 & 10.0 & 95 & 82 & 0 & 1.0 & 0.0 & 14.8 & 16.0 \\
\hline 30.12 .2011 & 10.47 & 14.0 & 10.4 & 97 & 82 & 0 & 1.0 & 0.0 & 14.8 & 16.0 \\
\hline 31.12 .2011 & 10.88 & 16.2 & 6.5 & 86 & 69 & 0 & 1.0 & 0.0 & 11.4 & 18.0 \\
\hline 1.01 .2012 & 11.07 & 17.8 & 5.6 & 89 & 78 & 1.5 & 0.8 & 0.0 & 9.8 & 19.0 \\
\hline 2.01 .2012 & 11.36 & 18.6 & 6.6 & 78 & 78 & 0 & 0.8 & 0 & 9.6 & 18.0 \\
\hline 3.01 .2012 & 12.43 & 19.2 & 6.2 & 81 & 75 & 0 & 0.8 & 0 & 9.0 & 18.5 \\
\hline 4.01 .2012 & 13.87 & 21.8 & 6.6 & 91 & 56 & 6.1 & 0.8 & 0 & 8.8 & 17.4 \\
\hline 5.01 .2012 & 13.99 & 23.8 & 5.6 & 78 & 40 & 6.7 & 0.8 & 0 & 10. & 19.6 \\
\hline 6.01 .2012 & 14.33 & 20.3 & 9.0 & 93 & 61 & 3.7 & 0.8 & 0 & 12.0 & 19.6 \\
\hline 7.01 .2012 & 15.43 & 21.8 & 10.2 & 97 & 68 & 1.0 & 0.8 & 0 & 13.0 & 19.0 \\
\hline 8.01 .2012 & 15.99 & 16.0 & 8.0 & 94 & 68 & 0 & 0.8 & 0 & 12.8 & 18.0 \\
\hline 9.01 .2012 & 16.22 & 17.2 & 7.6 & 87 & 62 & 0 & 0.8 & 0 & 12.0 & 17.0 \\
\hline 10.01 .2012 & 16.97 & 18.0 & 7.4 & 94 & 60 & 0 & 0.8 & 00 & 11.0 & 18.0 \\
\hline 11.01 .2012 & 17.23 & 18.2 & 4.6 & 85 & 56 & 0 & 0.8 & 0 & 8.6 & 18.0 \\
\hline 12.01 .2012 & 18.04 & 20.4 & 4.0 & 94 & 45 & 7.5 & 0.8 & 0 & 9.4 & 18.0 \\
\hline 13.01 .2012 & 18.98 & 21.2 & 4.4 & 87 & 79 & 0 & 0.8 & 0 & 9.4 & 18.0 \\
\hline 14.01 .2012 & 19.12 & 23.0 & 4.8 & 94 & 33 & 6.5 & 0.8 & 0 & 10.6 & 19.5 \\
\hline 15.01 .2012 & 20.23 & 22.0 & 7.6 & 94 & 39 & 8.5 & 1.2 & 0 & 9.6 & 19.5 \\
\hline 16.01 .2012 & 20.93 & 23.4 & 5.5 & 91 & 76 & 7.5 & 1.2 & 0 & 11.6 & 20.5 \\
\hline 17.01 .2012 & 21.47 & 21.2 & 6.2 & 94 & 72 & 6.4 & 1.2 & 0 & 11.2 & 20.5 \\
\hline 18.01 .2012 & 22.54 & 22.0 & 7.6 & 94 & 55 & 5.5 & 1.2 & 0 & 10.6 & 20.0 \\
\hline 19.01 .2012 & 23.68 & 21.0 & 4.6 & 97 & 63 & 5.5 & 1.2 & 0 & 10.5 & 20.6 \\
\hline 20.01 .2012 & 24.19 & 21.0 & 4.6 & 94 & 61 & 4.4 & 1.2 & 0 & 9.4 & 20.8 \\
\hline 21.01 .2012 & 25.43 & 21.4 & 5.6 & 97 & 50 & 6.1 & 1.2 & 0 & 9.4 & 20.2 \\
\hline 22.01 .2012 & 26.95 & 21.4 & 6.4 & 83 & 52 & 3.0 & 1.2 & 0 & 9.4 & 14.4 \\
\hline 23.01 .2012 & 27.86 & 22.0 & 7.4 & 81 & 48 & 6.9 & 1.2 & 0 & 10.0 & 14.4 \\
\hline 24.01 .2012 & 28.20 & 23.0 & 5.6 & 88 & 54 & 6.9 & 1.2 & 0 & 10.1 & 14.4 \\
\hline
\end{tabular}


Table.2 Disease severity of early blight of potato (Alternaria solani) during 2012-2013 at Kanpur

\begin{tabular}{|c|c|c|c|c|c|c|c|c|c|c|}
\hline \multirow[t]{2}{*}{ Date } & \multirow{2}{*}{$\begin{array}{l}\text { Disease } \\
\text { severity }(\%)\end{array}$} & \multicolumn{2}{|c|}{ Temp. } & \multicolumn{2}{|c|}{ RH. (\%) } & \multirow[b]{2}{*}{$\begin{array}{l}\text { Sun } \\
\text { Shine }\end{array}$} & \multirow[b]{2}{*}{ Evaporation } & \multirow[b]{2}{*}{ Rainfall } & \multicolumn{2}{|c|}{ Soil Temp. } \\
\hline & & Max & Min & Max & Min & & & & Max & Min \\
\hline 14.12 .2012 & 0.14 & 17.6 & 7.8 & 97 & 79 & 0 & 0.8 & 0 & 12.0 & 18.0 \\
\hline 15.12 .2012 & 0.22 & 12.6 & 7.2 & 88 & 78 & 0 & 0.8 & 0 & 12.5 & 20.0 \\
\hline 16.12 .2012 & 0.54 & 15.0 & 3.8 & 90 & 76 & 0 & 1.0 & 0 & 12.6 & 14.5 \\
\hline 17.12 .2012 & 0.76 & 16.6 & 3.8 & 100 & 87 & 0 & 0.8 & 0 & 11.8 & 16.0 \\
\hline 18.12 .2012 & 1.98 & 10.8 & 2.8 & 100 & 85 & 0 & 0.6 & 0 & 11.5 & 20.0 \\
\hline 19.12 .2012 & 2.32 & 12.4 & 2.0 & 100 & 68 & 0 & 1.0 & 0 & 9.5 & 17.0 \\
\hline 20.12 .2012 & 2.60 & 18.4 & 2.2 & 97 & 81 & 6.0 & 1.0 & 0 & 9.5 & 22.0 \\
\hline 21.12 .2012 & 3.13 & 17.4 & 5.6 & 97 & 59 & 0 & 1.2 & 0 & 7.5 & 23.0 \\
\hline 22.12 .2012 & 3.89 & 19.6 & 3.2 & 100 & 53 & 5.2 & 1.0 & 0 & 8.5 & 21.0 \\
\hline 23.12 .2012 & 4.12 & 17.6 & 4.0 & 100 & 54 & 5.0 & 0.8 & 0 & 10.0 & 22.2 \\
\hline 24.12 .2012 & 4.57 & 12.8 & 3.87 & 80 & 68 & 2.0 & 0.8 & 0 & 10.5 & 17.0 \\
\hline 25.12 .2012 & 4.99 & 11.0 & 1.4 & 100 & 85 & 00 & 0.8 & 0 & 9.0 & 14.0 \\
\hline 26.12 .2012 & 5.11 & 12.6 & 2.4 & 100 & 76 & 02.8 & 0.6 & 0 & 9.2 & 17.0 \\
\hline 27.12 .2012 & 5.20 & 13.4 & 3.8 & 94 & 64 & 0 & 0.6 & 0 & 9.5 & 17.5 \\
\hline 28.12 .2012 & 5.97 & 13.0 & 2.0 & 93 & 68 & 0 & 0.6 & 0 & 8.5 & 16.6 \\
\hline 29.12 .2012 & 6.13 & 16.4 & 1.1 & 100 & 54 & 3.0 & 0.6 & 0 & 6.5 & 17.5 \\
\hline 30.12 .2012 & 6.54 & 19.0 & 0.4 & 96 & 43 & 6.0 & 0.6 & 0 & 6.0 & 19.0 \\
\hline 31.12 .2012 & 6.87 & 20.4 & 3.8 & 78 & 46 & 7.2 & 1.0 & 0 & 8.0 & 20.5 \\
\hline 1.01 .2013 & 7.03 & 21.8 & 4.8 & 74 & 44 & 8.5 & 1.0 & 0 & 9.0 & 22.0 \\
\hline 2.01 .2013 & 7.28 & 21.4 & 4.7 & 100 & 55 & 0 & 1.0 & 0 & 9.0 & 21.0 \\
\hline 3.01 .2013 & 7.46 & 25.6 & 6.7 & 85 & 51 & 0.2 & 1.0 & 0 & 11.0 & 24.0 \\
\hline 4.01 .2013 & 7.85 & 32.0 & 7.7 & 97 & 68 & 0 & 1.0 & 0 & 11.5 & 23.0 \\
\hline 5.01 .2013 & 7.95 & 23.6 & 6.8 & 100 & 58 & 0 & 1.0 & 0 & 12.0 & 23.0 \\
\hline 6.01 .2013 & 8.00 & 24.8 & 11.4 & 89 & 60 & 0 & 1.0 & 0 & 13.5 & 23.5 \\
\hline 7.01 .2013 & 8.10 & 32.8 & 12.4 & 87 & 59 & 0 & 1.0 & 0 & 15.0 & 20.2 \\
\hline 8.01 .2013 & 8.40 & 25.4 & 12.7 & 91 & 74 & 3.0 & 1.0 & 3.5 & 14.5 & 20.0 \\
\hline 9.01 .2013 & 10.35 & 19.8 & 9.4 & 95 & 56 & 8.4 & 1.0 & 1.1 & 12.0 & 22.2 \\
\hline 10.01 .2013 & 10.59 & 19.4 & 7.6 & 92 & 59 & 8.9 & 1.0 & 0 & 10.2 & 22.4 \\
\hline 11.01 .2013 & 10.99 & 16.6 & 5.4 & 100 & 81 & 4.9 & 1.0 & 0 & 11.0 & 21.0 \\
\hline 12.01 .2013 & 11.05 & 16.0 & 4.7 & 100 & 75 & 3.5 & 0.5 & 0 & 11.0 & 19.2 \\
\hline 13.01 .2013 & 11.36 & 15.8 & 5.7 & 100 & 87 & 1.3 & 0.5 & 0 & 11.0 & 18.0 \\
\hline 14.01 .2013 & 11.75 & 17.8 & 4.8 & 97 & 67 & 5.1 & 0.5 & 0 & 11.0 & 21.2 \\
\hline 15.01 .2013 & 12.06 & 21.4 & 5.6 & 92 & 52 & 8.6 & 1.0 & 0 & 8.6 & 22.5 \\
\hline 16.01 .2013 & 12.54 & 20.6 & 6.4 & 84 & 48 & 9.0 & 1.0 & 0 & 11.0 & 22.6 \\
\hline 17.01 .2013 & 12.82 & 21.8 & 6.8 & 80 & 50 & 8.9 & 1.0 & 0 & 10.5 & 23.4 \\
\hline 18.01 .2013 & 13.36 & 21.2 & 3.6 & 91 & 49 & 6.3 & 1.0 & 0 & 9.0 & 23.0 \\
\hline 19.01 .2013 & 13.59 & 22.0 & 4.4 & 91 & 54 & 6.1 & 1.0 & 0 & 9.5 & 22.8 \\
\hline 20.01 .2013 & 14.06 & 22.4 & 5.6 & 97 & 64 & 4.4 & 1.0 & 0 & 10.0 & 22.5 \\
\hline 21.01.2013 & 14.18 & 22.4 & 4.8 & 91 & 58 & 4.2 & 1.0 & 0 & 10.5 & 23.0 \\
\hline 22.01 .2013 & 14.78 & 24.4 & 6.08 & 92 & 45 & 3.7 & 1.2 & 0 & 11.2 & 24.0 \\
\hline 23.01 .2013 & 15.06 & 24.8 & 8.8 & 90 & 62 & 7.0 & 1.2 & 0 & 13.0 & 22.5 \\
\hline
\end{tabular}


Table.3 Disease severity of Early blight of potato (Alternaria solani) during 2011-2012 at Faizabad

\begin{tabular}{|c|c|c|c|c|c|c|c|c|c|c|}
\hline \multirow[t]{2}{*}{ Date } & \multirow{2}{*}{$\begin{array}{l}\text { Disease } \\
\text { severity } \\
(\%)\end{array}$} & \multicolumn{2}{|c|}{ Temp. } & \multicolumn{2}{|c|}{ RH. (\%) } & \multirow[b]{2}{*}{$\begin{array}{l}\text { Sun } \\
\text { Shine }\end{array}$} & \multirow[b]{2}{*}{ Evaporation } & \multirow[b]{2}{*}{ Rainfall } & \multicolumn{2}{|c|}{ Soil Temp. } \\
\hline & & $\operatorname{Max}$ & Min & $\begin{array}{l}\text { Ma } \\
\mathbf{x}\end{array}$ & Min & & & & $\operatorname{Max}$ & Min \\
\hline 18.12 .2011 & 0.42 & 23.5 & 6 & 88 & 46 & 7 & 1.6 & 0 & 12.5 & 19.0 \\
\hline 19.12.2011 & 0.56 & 24.5 & 6.5 & 78 & 35 & 7.5 & 1.8 & 0 & 11.5 & 24.0 \\
\hline 20.12 .2011 & 0.89 & 25.5 & 6.5 & 88 & 47 & 7.5 & 1.2 & 0 & 11.5 & 19. \\
\hline 21.12 .2011 & 1.25 & 25 & 6.5 & 78 & 56 & 5 & 1. & 0 & 13.0 & 19.0 \\
\hline 22.12 .2011 & 1.49 & \begin{tabular}{|l|}
19.5 \\
\end{tabular} & 13.5 & 98 & 76 & 0 & 1.2 & 0 & 15.5 & 18.0 \\
\hline 23.12 .2011 & 1.99 & 17.9 & 11.5 & 91 & 90 & 0 & 0.6 & 0 & 14.0 & 17.0 \\
\hline 24.12 .2011 & 3.98 & 21 & 13.5 & 95 & 81 & 4 & 1.6 & 0 & 14.5 & 18.5 \\
\hline 25.12 .2011 & 4.72 & 22 & 10.5 & 86 & 65 & 4 & 1.6 & 0 & 13.5 & 25.0 \\
\hline 26.12 .2011 & 5.60 & 17 & 10.5 & 83 & 88 & 2 & 1.4 & 0 & 14.0 & 18.0 \\
\hline 27.12 .2011 & 6.46 & 18 & 9.5 & 97 & 62 & 2 & 1.8 & 0 & 13.0 & 17.0 \\
\hline 28.12 .2011 & 7.22 & 20.5 & 13.5 & 89 & 81 & 0 & 1.7 & 0 & 14.5 & 17.5 \\
\hline 29.12 .2011 & 7.22 & 16 & 11.5 & 97 & 89 & 0 & 1.6 & 0 & 13.0 & 17.0 \\
\hline 30.12 .2011 & 8.01 & 13.5 & 11 & 91 & 88 & 0 & 1.7 & 0 & 15. & 15.5 \\
\hline 31.12 .2011 & 8.24 & 16 & 9.5 & 97 & 78 & 0 & 1.6 & 0 & 13.0 & 14.0 \\
\hline 1.01 .2012 & 8.50 & 19 & 6.5 & 100 & 62 & 0 & 1.8 & 0 & 12.0 & 16.5 \\
\hline 2.01 .2012 & 11.50 & 20 & 5.5 & 92 & 62 & 2.5 & 1.6 & 0 & 10.5 & 16.0 \\
\hline 3.01 .2012 & 12.10 & 20 & 6 & 87 & 56 & 7 & 1.8 & 0 & 09.5 & 18.0 \\
\hline 4.01 .2012 & 12.92 & 22.5 & 6 & 87 & 59 & 7 & 2.2 & 0 & 08.5 & 19.5 \\
\hline 5.01 .2012 & 13.10 & 21 & 6 & 90 & 72 & 7.5 & 2.2 & 0 & 09.5 & 20. \\
\hline 6.01 .2012 & 14.87 & 21.5 & 10.5 & 84 & 57 & 2 & 2.2 & 0 & 12.5 & 25.5 \\
\hline 7.01 .2012 & 15.63 & 22 & 9.5 & 91 & 65 & 2 & 1.6 & 0 & 13.5 & 19.0 \\
\hline 8.01 .2012 & 16.44 & 21 & 9.5 & 98 & 64 & 2 & 2.0 & 0 & 12.5 & 18.5 \\
\hline 9.01 .2012 & 17.52 & 20.5 & 9 & 88 & 72 & 1.5 & 2.2 & 0 & 12.0 & 18.0 \\
\hline 10.01 .2012 & 17.96 & 18.5 & 5.5 & 90 & 59 & 5 & 2.0 & 0 & 11.5 & 19.5 \\
\hline 11.01 .2012 & 18.25 & 20 & 5 & 90 & 72 & 7.5 & 2.4 & 0 & 09.5 & 15.5 \\
\hline 12.01 .2012 & 20.42 & 21 & 5 & 87 & 49 & 7.5 & 2.6 & 0 & 09.5 & 20.5 \\
\hline 13.01 .2012 & 21.86 & 22.5 & 6.5 & 90 & 66 & 8 & 3.6 & 0 & 10.0 & 19.0 \\
\hline 14.01 .2012 & 22.98 & 24 & 5 & 87 & 59 & 7.5 & 3.4 & 0 & 10.5 & 21.5 \\
\hline 15.01 .2012 & 23.03 & 23.5 & 6 & 92 & 45 & 7 & 3.0 & 0 & 11.5 & 18.0 \\
\hline 16.01 .2012 & 23.42 & 24 & 5 & 87 & 59 & 4 & 2.2 & 0 & 10.5 & 19.0 \\
\hline 17.01 .2012 & 24.52 & 23.5 & 6 & 88 & 45 & 7 & 1.6 & 0 & 10.5 & 20.0 \\
\hline 18.01 .2012 & 25.84 & 24 & 5 & 88 & 59 & 8 & 2.4 & 0 & 11.0 & 20.0 \\
\hline 19.01 .2012 & 26.13 & 22 & 5 & 67 & 59 & 8.5 & 2.2 & 0 & 11.0 & 18.0 \\
\hline 20.01 .2012 & 26.98 & 22.5 & 4.5 & 87 & 43 & 8 & 2.4 & 0 & 11.5 & 18.5 \\
\hline 21.01 .2012 & 27.15 & 22 & 5 & 97 & 63 & 7.5 & 2.2 & 0 & 10.0 & 20.0 \\
\hline 22.01 .2012 & 28.02 & 22 & 4.5 & 84 & 72 & 8.0 & 2.2 & 0 & 10.0 & 22.0 \\
\hline 23.01 .2012 & 28.89 & 22.5 & 4.4 & 74 & 62 & 7.5 & 2.4 & 0 & 10.0 & 18.0 \\
\hline 24.01 .2012 & 29.13 & 24 & 5 & 90 & 59 & 7 & 2.0 & 0 & 10.5 & 18.5 \\
\hline
\end{tabular}


Table.4 Disease severity of Early blight of potato (Alternaria solani) during 2012-2013 at Faizabad

\begin{tabular}{|c|c|c|c|c|c|c|c|c|c|c|}
\hline \multirow[t]{2}{*}{ Date } & \multirow{2}{*}{$\begin{array}{l}\text { Disease } \\
\text { severity } \\
(\%)\end{array}$} & \multicolumn{2}{|c|}{ Temp. } & \multicolumn{2}{|c|}{ RH. (\%) } & \multirow[b]{2}{*}{$\begin{array}{l}\text { Sun } \\
\text { Shine }\end{array}$} & \multirow[b]{2}{*}{ Evaporation } & \multirow[b]{2}{*}{ Rainfall } & \multicolumn{2}{|c|}{ Soil Temp. } \\
\hline & & Max & Min & Max & Min & & & & Max & Min \\
\hline 16.12 .2012 & 0.23 & 23.4 & 7.8 & 98 & 67 & 0 & 0.9 & 0 & 13.5 & 15.0 \\
\hline 17.12 .2012 & 0.45 & 23.5 & 7.6 & 100 & 77 & 0 & 0.8 & 0 & 10.0 & 15.0 \\
\hline 18.12 .2012 & 0.63 & 23.4 & 7.1 & 100 & 86 & 0 & 1.4 & 0 & 09.0 & 14.0 \\
\hline 19.12 .2012 & 0.79 & 25.0 & 7.7 & 100 & 88 & 4.0 & 1.4 & 0 & 09.0 & 14.0 \\
\hline 20.12 .2012 & 1.18 & 25.0 & 7.3 & 100 & 53 & 0 & 1.2 & 0 & 09.5 & 17.0 \\
\hline 21.12 .2012 & 1.56 & 25.0 & 7.5 & 78 & 77 & 5.0 & 1.2 & 0 & 10.0 & 15.0 \\
\hline 22.12 .2012 & 2.33 & 21.5 & 5.5 & 92 & 49 & 5 & 1.7 & 0 & 10.0 & 21.0 \\
\hline 23.12 .2012 & 3.16 & 22.5 & 6.0 & 89 & 45 & 4 & 1.6 & 0 & 11.0 & 21.0 \\
\hline 24.12 .2012 & 4.42 & 14.0 & 4.0 & 87 & 67 & 2 & 1.8 & 0 & 12.0 & 16.0 \\
\hline 25.12 .2012 & 5.67 & 12.0 & 3.5 & 100 & 76 & 0 & 1.4 & 0 & 10.5 & 15.0 \\
\hline 26.12.2012 & 6.98 & 9.5 & 3.0 & 100 & 97 & 0 & 1.1 & 0 & 09.0 & 13.0 \\
\hline 27.12 .2012 & 8.56 & 11.5 & 4.5 & 94 & 88 & 0 & 0.7 & 0 & 10.0 & 12.0 \\
\hline 28.12 .2012 & 9.12 & 9.0 & 4.0 & 97 & 70 & 0 & 0.9 & 0 & 08.5 & 11.0 \\
\hline 29.12 .2012 & 10.14 & 12.5 & 0.4 & 94 & 59 & 0 & 1.1 & 0 & 07.0 & 14.0 \\
\hline 30.12 .2012 & 11.41 & 15.0 & 0.3 & 100 & 58 & 0 & 1.2 & 0 & 08.0 & 16.0 \\
\hline 31.12 .2012 & 11.81 & 19.5 & 0.2 & 100 & 46 & 1.0 & 1.3 & 0 & 10. & 21.0 \\
\hline 1.01 .2013 & 12.32 & 17.5 & 1.4 & 97 & 54 & 1.5 & 1.6 & 0 & 11.0 & 21.0 \\
\hline 1.01 .2013 & 12.99 & 20.5 & 4.0 & 100 & 53 & 2.5 & 1.4 & 0 & 10.5 & 21.5 \\
\hline 2.01 .2013 & 13.76 & 25.0 & 5.5 & 87 & 41 & 4.0 & 1.5 & 0 & 09.5 & 13.0 \\
\hline 3.01 .2013 & 14.11 & 25.0 & 7.0 & 97 & 39 & 5.0 & 1.82 .0 & 0 & 12.0 & 16.5 \\
\hline 4.01 .2013 & 14.96 & 24.0 & 6.5 & 89 & 45 & 5.0 & 1.8 & 0 & 11.5 & 22.5 \\
\hline 5.01 .2013 & 15.38 & 25.0 & 9.5 & 89 & 39 & 1.0 & 2.0 & 0 & 14.0 & 23.0 \\
\hline 6.01 .2013 & 16.43 & 24.0 & 9.5 & 89 & 61 & 0 & 1.8 & 00 & 15.0 & 21.0 \\
\hline 7.01 .2013 & 17.64 & 23.0 & 1.4 & 93 & 67 & 0 & 2.0 & 0 & 15.0 & 22.0 \\
\hline 8.01 .2013 & 21.11 & 20.5 & 1.3 & 91 & 53 & 0 & 1.4 & 3.2 & 15.0 & 21.0 \\
\hline 9.01 .2013 & 21.40 & 20.5 & 8 & 97 & 44 & 6.5 & 1.6 & 0 & 10.0 & 21.0 \\
\hline 10.01 .2013 & 22.06 & 18.0 & 7 & 89 & 43 & 4.0 & 1.8 & 0 & 12.0 & 20.0 \\
\hline 11.01 .2013 & 22.47 & 17 & 7 & 97 & 88 & 3.0 & 1.6 & 0 & 12.0 & 19.0 \\
\hline 12.01 .2013 & 22.87 & 14 & 6 & 92 & 67 & 2.0 & 1.4 & 0 & 10.0 & 18.0 \\
\hline 13.01 .2013 & 22.92 & 12 & 5 & 97 & 76 & 0 & 1.6 & 0 & 10.0 & 14.0 \\
\hline 14.01 .2013 & 23.09 & 16.5 & 5.5 & 100 & 58 & 2.0 & 1.5 & 0 & 10.0 & 18.0 \\
\hline 15.01 .2013 & 26.55 & 21 & 5.0 & 100 & 45 & 5.5 & 1.5 & 0 & 10.5 & 21.0 \\
\hline 16.01 .2013 & 27.44 & 21 & 2.5 & 100 & 49 & 5.0 & 1.6 & 0 & 10.0 & 22.0 \\
\hline 17.01 .2013 & 28.39 & 21.5 & 4.0 & 87 & 45 & 5.0 & 2.0 & 0 & 11.0 & 22.5 \\
\hline 18.01 .2013 & 29.18 & 22 & 4.5 & 100 & 45 & 5.0 & 2.0 & 0 & 10.5 & 22.0 \\
\hline 19.01.2013 & 30.17 & 23 & 2.5 & 97 & 58 & 5.0 & 1.8 & 0 & 10.0 & 23.0 \\
\hline 20.01 .2013 & 30.98 & 24 & 4.5 & 97 & 61 & 6.0 & 2.0 & 0 & 10.0 & 24.0 \\
\hline 21.01 .2013 & 31.23 & 25 & 7 & 89 & 38 & 7 & 2.2 & 0 & 11.0 & 24.0 \\
\hline
\end{tabular}


Table.5 Correlation between disease severity with temperature, RH\%, Sunshine hours at Kanpur 2011-2012

\begin{tabular}{|l|l|l|l|l|l|l|}
\hline & $\begin{array}{l}\text { Disease } \\
\text { severity } \\
(\%)\end{array}$ & $\begin{array}{l}\text { Temp } \\
\text { max. }\end{array}$ & $\begin{array}{l}\text { Temp } \\
\text { min. }\end{array}$ & RH max. & RH min. & $\begin{array}{l}\text { Sun } \\
\text { shine }\end{array}$ \\
\hline Dsp & 1 & & & & & \\
\hline Temp max & 0.141015 & 1 & & & & \\
\hline Temp min & -0.17845 & -0.51214 & 1 & & & \\
\hline RH max & -0.3355 & -0.09036 & 0.278203 & 1 & & \\
\hline RH min & -0.16499 & -0.69389 & 0.638215 & 0.149054 & 1 & \\
\hline Sun shine & 0.366387 & 0.720023 & -0.49268 & -0.04185 & -0.70934 & 1 \\
\hline & & & & & & \\
\hline
\end{tabular}

Table.6 Correlation between disease severity to temperature, RH\%, Sunshine hours at Kanpur 2012-2013

\begin{tabular}{|l|l|l|l|l|l|l|}
\hline & Dsp & $\begin{array}{l}\text { Temp } \\
\text { max. }\end{array}$ & $\begin{array}{l}\text { Temp } \\
\text { min. }\end{array}$ & RH max. & $\begin{array}{l}\text { RH } \\
\text { min. }\end{array}$ & $\begin{array}{l}\text { Sun } \\
\text { shine }\end{array}$ \\
\hline Dsp & 1 & & & & & \\
\hline $\begin{array}{l}\text { Temp } \\
\text { max }\end{array}$ & 0.519258 & 1 & & & & \\
\hline $\begin{array}{l}\text { Temp } \\
\text { min }\end{array}$ & 0.318911 & 0.662035 & 1 & & & \\
\hline RH max & -0.20731 & -0.30333 & -0.29188 & 1 & & \\
\hline RH min & -0.43293 & -0.49168 & -0.0617 & 0.476215 & 1 & \\
\hline Sun shine & 0.554468 & 0.192925 & 0.009051 & -0.37277 & -0.5233 & 1 \\
\hline
\end{tabular}

Table.7 Correlation between disease severity with temperature, $\mathrm{RH} \%$, sunshine hours at Faizabad during 2011-2012

\begin{tabular}{|l|c|c|c|c|c|c|}
\hline & Dsp & $\begin{array}{c}\text { Temp } \\
\text { max. }\end{array}$ & $\begin{array}{c}\text { Temp } \\
\text { min. }\end{array}$ & RH max. & RH min. & $\begin{array}{c}\text { Sun } \\
\text { shine }\end{array}$ \\
\hline Dsp & 1 & & & & & \\
\hline Temp max & 0.20812 & 1 & & & & \\
\hline Temp min & -0.20813 & -0.32455 & 1 & & & \\
\hline RH max & -0.26344 & -0.00971 & 0.248176 & 1 & & \\
\hline RH min & -0.42618 & -0.53674 & 0.804576 & 0.350474 & 1 & \\
\hline Sun shine & 0.27322 & 0.540208 & -0.59335 & -0.3411 & -0.61419 & 1 \\
\hline
\end{tabular}


Table.8 Correlation between disease severity to temperature, $\mathrm{RH} \%$, Sunshine hours at Faizabad 2012-2013

\begin{tabular}{|c|c|c|c|c|c|c|}
\hline & Dsp & $\begin{array}{c}\text { Temp } \\
\text { max. }\end{array}$ & $\begin{array}{c}\text { Temp } \\
\text { min. }\end{array}$ & RH max. & $\begin{array}{c}\text { RH } \\
\text { min. }\end{array}$ & $\begin{array}{c}\text { Sun } \\
\text { shine }\end{array}$ \\
\hline Dsp & 1 & & & & & \\
\hline $\begin{array}{c}\text { Temp } \\
\text { max }\end{array}$ & 0.050787 & 1 & & & & \\
\hline $\begin{array}{c}\text { Temp } \\
\text { min }\end{array}$ & -0.15587 & 0.463563 & 1 & & & \\
\hline RH max & -0.00569 & -0.22408 & 0.24917 & 1 & & \\
\hline RH min & -0.42169 & -0.44179 & 0.00531 & 0.229462 & 1 & \\
\hline Sunshine & 0.488436 & 0.471475 & 0.23057 & -0.25609 & 0.50429 & 1 \\
\hline
\end{tabular}

Fig.1 Disease severity curve correlated with Temperature, Relative humidity, and Sunshine hour at Kanpur during 2011-12

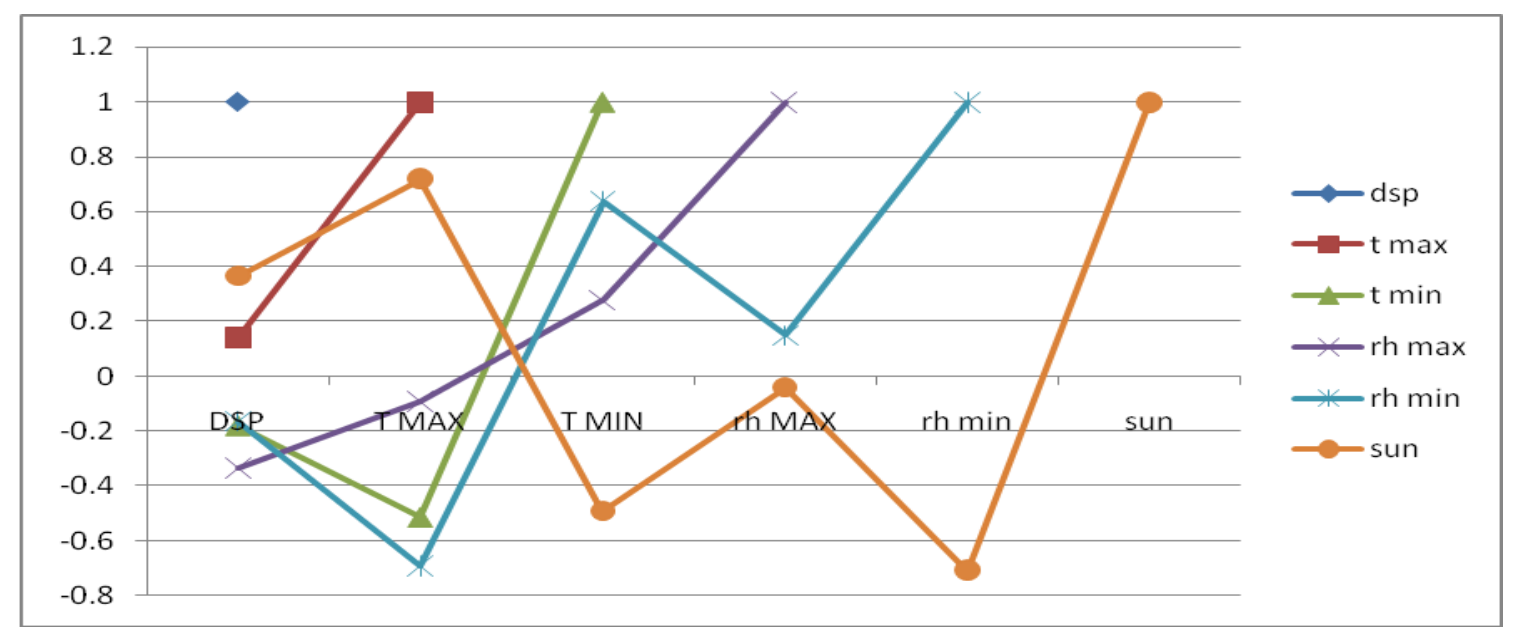

Fig.2 Disease severity curve correlated with Temperature, Relative humidity, and Sunshine hour at Kanpur during 2012-13

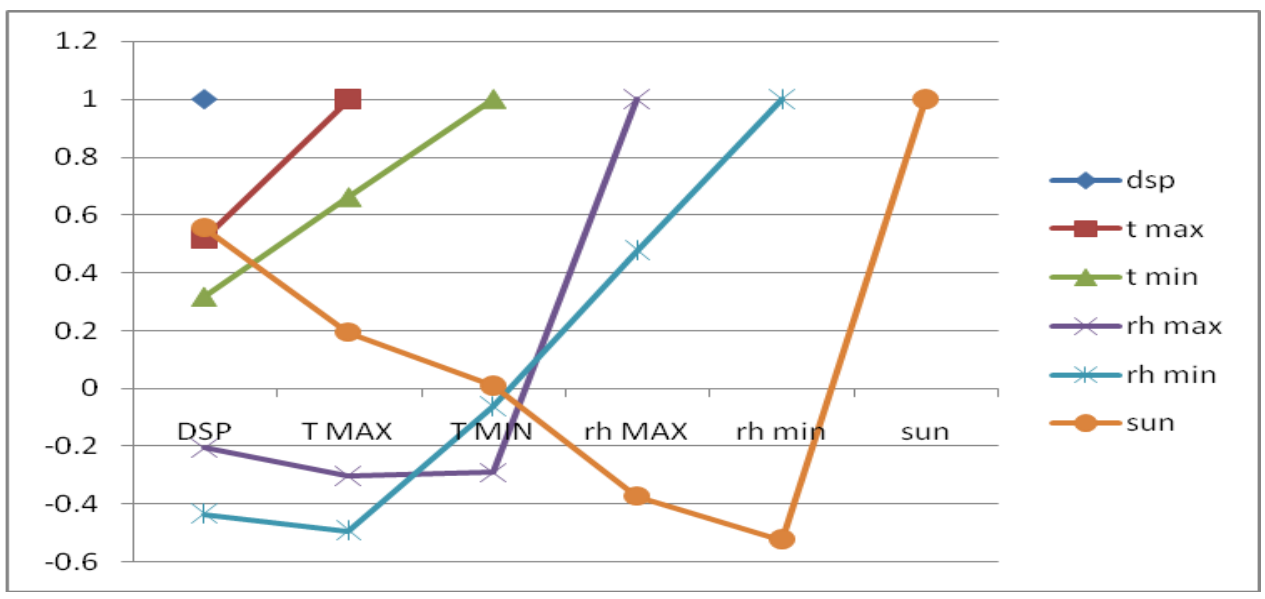


Fig.3 Disease severity curve correlated with temperature, relative humidity, and sunshine hour at Faizabad during 2011-12

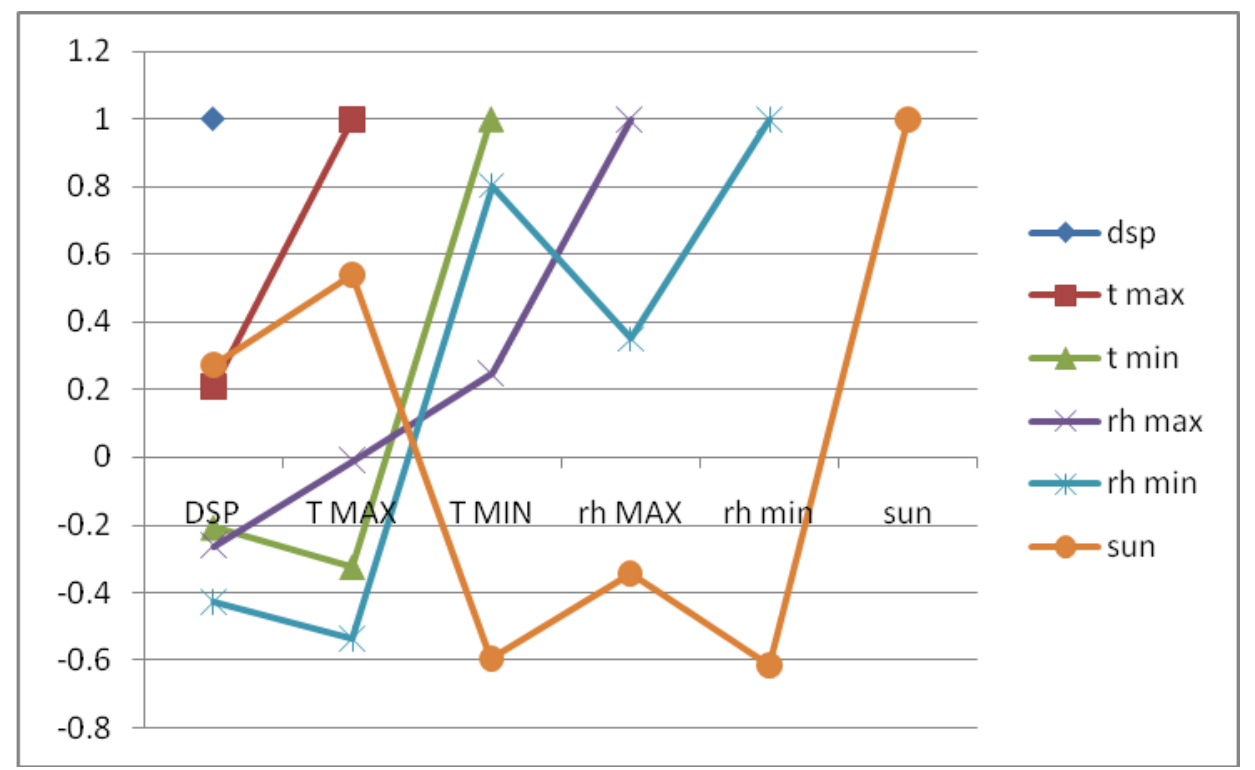

Fig.4 Disease severity curve correlated with temperature, relative humidity, and sunshine hour at Faizabad during 2012-13

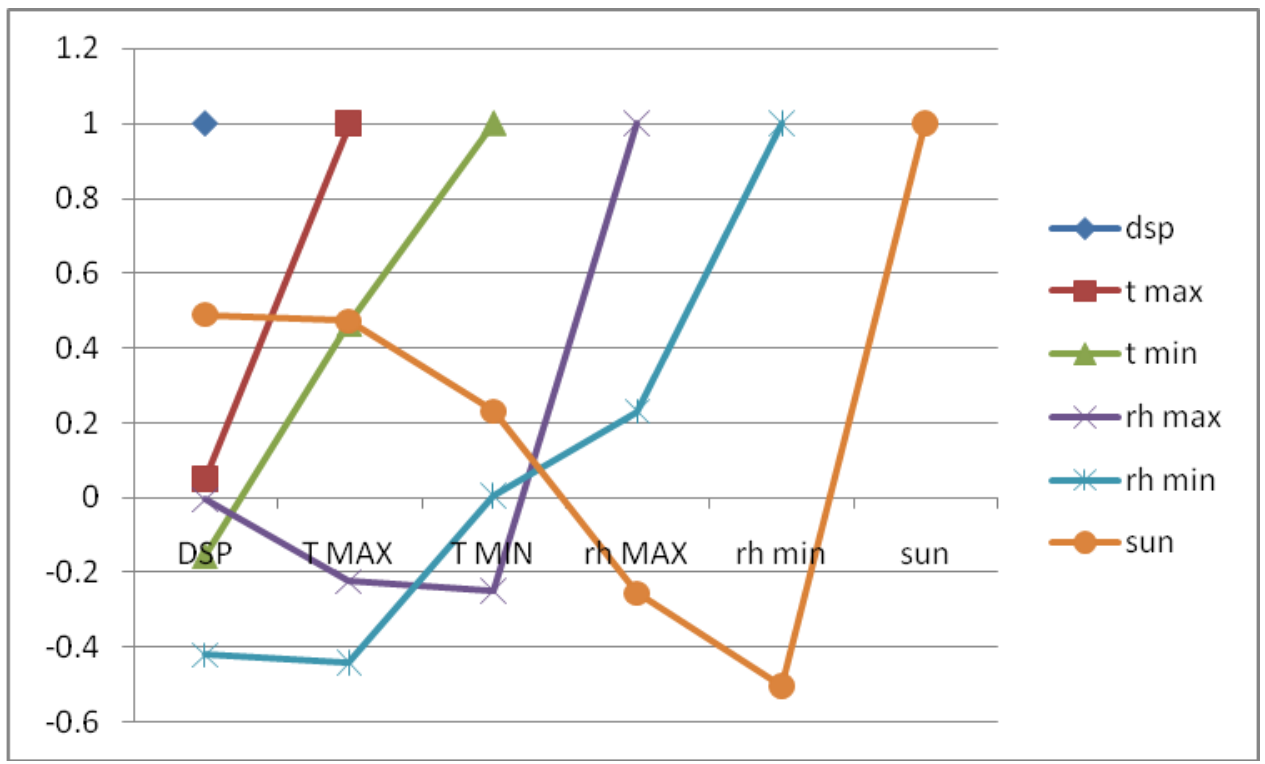

The spore content in the atmosphere increases when the mean, maximum and minimum temperatures increases, making Alternaria a temperature-dependent fungus. Hjelmroos, (1993) reported that Alternaria is a saprophytic genus with an optimal development shown to occur in the temperature ranges of $22-28^{\circ} \mathrm{C}$.
The co-relation between disease severity with temperature, relative humidity and sunshine hours were calculated by standard statistical calculation and the results showed that disease severity co-relate negatively with temperature and sunshine hours, representing the value (-0.3984) and (-0.4509), respectively, but positively co-relate with 
relative humidity (0.5814) (Biswas et al., 2013 ).

Angulo-Romero et al., (1999) found that the most conidia appear in the atmosphere when minimum temperatures are over $10^{\circ} \mathrm{C}$, maximum temperatures are under $30^{\circ} \mathrm{C}$ and mean temperatures are between 20 and $25^{\circ} \mathrm{C}$.

\section{References}

Adams, S.S. and Stevenson, W.R. 1990. Water management, disease development and potato production. Amri. Potato J., 67: 311.

Angulo-Romero, J. Mediavilla-Molina, A. and Domínguez-Vilches, E. 1999. Conidia of Alternaria in the atmosphere of the city of Córdoba, Spain in relation to meteorological parameters. Int. J. Biometeorol., 43: 45-49.

Biswas, S.K., Ajay Rawat and Praveen Kumar. 2013. Epidemiological studies of late blight of potato [Phytophthora infestans (Mont.) be Berry] and its correlation with disease severity. J. Mycopathol. Res., 51(2): 235-242.

Christ, B.J. and Maczuga, S.A. 1989. The effect of fungicide schedules and inoculums levels on early blight severity and yield of potato. Plant Dis., 73(8): 695-698.

Harrison, M.D., Livingston, C.H. and Oshima, N. 1965. Epidemiology of potato early blight in Colorado: 1. Initial infection, disease development and the influence of the environmental factors. Amri. Pot. J., 42: 279-291.

Hjelmroos, M. 1993. Relationship between airborne fungal spore presence and weather variables. Grana., 32: 40-47.

Malcolimson, J.F. 1976. Assessment of field resistance to late blight (Phytophthora infestans) in potatoes. Trans. Br. Mycol. Soc., 67: 321-325.

Nnodu, E.C., Harrison, M.D. and Parke, R.V. 1982. The effect of temperature and relative humidity on wound healing and infection of potato tubers by Alternaria solani. Amri. Pot. J., 59: 297-311.

Rotem, J. and Feldman, S. 1965. The relation between the ratio of yield to foliage and the incidence of early blight in potato and tomato. Israel J. Agric. Res., 15: 115122.

Singh, R.S. 2007. Plant Dis., $8^{\text {th }}$ ed., Oxford \& IBH Publishing Co. Pvt. Ltd., New Delhi. Pp. 169-170.

Shailbala and Pathak, C. 2008. Harnessing the potential of potato to meet increasing food demand. Kurukshetra, 56(3): 45-48.

Shtienberg, D. and Fry, W.E. 1990. Influence of host resistance and crop rotation on initial appearance of potato early blight. Plant Dis., 74: 849-852.

Troutt, C., Levetin, E. 2001. Correlation of spring spore concentrations and meteorological conditions in Tulsa, Oklahoma. Int. J. Biometeorol., 45(2): 64-74

Vander Walls, J.E., Korsen, L. and Aveling, T.A.S. 2001. A review of early blight of potato. Afr. Plant Protect, 70: 91-102.

Vloutoglou, I. and Kalogerakis, S.N. 2000. Effects of inoculum concentration, wetness duration and plant age on development of early blight (Alternaria solani) and on shedding of leaves in tomato plants. Plant Pathol., 49: 339-345.

\section{How to cite this article:}

Praveen Kumar, S.K. Biswas, Virendra Kumar and Kishan Lal. 2017. Epidemiological Studies on Early Blight of Potato under Climate Change and its Co-Relation with Disease Severity. Int.J.Curr.Microbiol.App.Sci. 6(5): 1725-1736. doi: https://doi.org/10.20546/ijcmas.2017.605.187 